\title{
Mobile Colored Overlays for People with Visual Stress
}

\author{
Young Gun Jang \\ Dept. of Computer and Information Engr. Chongju University, Korea \\ ygjang@cju.ac.kr
}

\begin{abstract}
A colored overlay has been used as an assistive device for people with pisual stress. Recently, a kind of mobile colored overlays has been developed; however, itcan be applied to all contents of its environment. In this paper, I implemented a mobile overlay application based on the Android operating system and it can be applied to not only all contents in the system but also external contents such as book using camera node, To achieve this, I measured film overlay colors at transmit mode and reflect mode by using spectrum colorimeter and estimated and implemented color information to apply to virtual overlays. Chromaticity measured in reflect mode was hearier than the one measured in transmit mode. I found I needed to use chromaticity measured in reflect mode in order to keep the film's overlay effect in virtual colored overlay, which was applied, to printed paper. The test results showed that it had the same effect as whenexisting film oyerlay was used on white paper, with the exception of color effect on the whe screen of mobile devices.
\end{abstract}

Keywords: Visual stress, colored overlays, mobile device, Android operating system, chromaticity in reflect mode

\section{Introduction}

In December 2008, W3C announced WCAG 2.0 [1] which contained the first specifications on seizure and was related to mobile accessibility. In December 2010, the Korea Communication Commission legislated the Korean Web Accessibility Guidelines 2.0 [2] as a standard, and in September 2011, the Ministry of Public Administration and Security announced the Mobile Application Accessibility Guidelines [3] as a notification. These regulations contained additional specifications on scotopic sensitivity seizure and introduced methods necessary for establishing web contents for those who had this symptom so that they could easily access information. These regulations were mandatory. However, test items were limited, not providing contents like flickering or blinking at 3-50 Hz frequency, and did not consider those who had light visual stress. Symptoms of visual stress are divided into visual discomfort such as blurring, floating, and zig-zag line crossing, and visual distortion such as dizziness and nausea. These can lead to reading disability, which in turn can develop as a learning disability. Another type of it is scotopic seizure, which can be more serious when a display device is smaller. For this reason, accessibility issues in mobile devices such as a smart phone or tablet PC, which are smaller display devices, can be more serious. According to a recent "analysis on usage of mobile video" conducted among 1,023 Korean Internet consumers by Youtube, the highest usage category was communication $(89.9 \mathrm{~min})$ followed by music listening and watching TV broadcasting contents. The rate of e-Book reading time significantly increased at up to $50 \mathrm{~min}$ a day whereas only an average of $6.1 \mathrm{~min}$ daily was used for reading on desktop PCs [4]. The usage of electrical textbook with the i-Pad or a 
similar type of tablet PC was replaced with paper textbook in CA, USA. This trend is expected to increase.

Olive Mears reported that children's awareness with books and their reading disability were affected by the features of printed materials. Reading disability could be reduced when the size of books was reduced, colored paper was used, and contrast was reduced [5]. Effectiveness of colored overlays to mitigate this symptom was first reported by $\mathrm{H}$. Irlen who first named this symptom as scotopic syndrome [6]. Irlen first invented a film type of color overlay applied empirically to those with visual stress from 1980 and its effect was proven. Color stimuli vary among subjects and their effect is greatly demonstrated in specific color stimuli. Virtual colored overlay that can be used on PC was first introduced in the world as Virtual Reading Ruler [7] and it was first developed in 2008 in Korea too [8]. Recently, a kind of virtual colored overlays in mobile device has been developed; however, it can't be applied to all contents of its environment $[9,10]$. Therefore, it is an urgent requirement to develop assistive devices that improve accessibility to electronic documents for those with paper aims to provide systematic color stimuli which are known to be effective for those with visual stress. It also purposes to implement virtual coldred overlay as a color stimulating device working among smart phones or tablet PCs based on the Android operating system, with the subjects being able to choose effective colors for themselves.

\section{Estimation of Color Information of Mobile Colored Overlay}

Designating colors is one of the most cruciat matters in the design of virtual colored overlay. For colored overlay frrm types, Irlen Overlays were used from the early 1980s and then Intuitive Overlays were used since 1995 . These two overlays were recognized for over a decade for their effectiveness, and color samples were empirically validated sufficiently. Irlen Overlays and Intuitive Overlays, two representative overlays, showed significant differences in chromatidity, but no correct chromaticity has been published. For color samples in this paper, real product was measured by reference to Intuitive Overlays [11].

With a spectrophotometer, 1 measured color information and transmissivity of a single color, overlap of a singte color, and overlap of a neighbor color of Intuitive Overlays with transmit mode. It was deeided that this experiment was useful to analyze the features of film overlay itself. The spectrophotometer used for measurement was the CM-3600d Konica Minolta brand The measurement method used involved compensation of the device with the overlay filmplaeed at the entrance of the receiver, lights penetrating the rougher side (visual side), and quantified overlay colors (CIE-L*u'v') and transmissivity. The chromaticities of measured overlays are presented in Figure 1(a) as CIE Lu'v' coordinate.

In opder to measure the features of film overlay with reflect mode, CM-2600d, a spectrophotometer by Konica Minolta, and stick film overlay for white A4 copy paper were used while chromaticity and reflection rate were measured. Applied features were meant to be visual features, which were transferred to users when film overlay was used in white paper books. In general, Wilkins Reading Rate Test used film overlays, and Visual Stress Assessment Pack of Crossbow Education also applied film overlay to white and ivory colors, respectively, for experiment about selecting optimized colors. Since CM2600d adhered closely to the object vertically due to its mechanical structure for measurement, it had the advantages of blocking out surrounding illumination and reducing chromaticity error caused by changing the measuring angle. Measured colors are presented in Figure 1(b) as CIE Lu'v' coordinate. 
I measured the chromaticity of film overlay by using the Galaxy Tab 10.1, a tablet PC on the Android mobile platform. For measuring chromaticity, I used CS-200 portable Chroma meter by Konica Minolta. CS-200 could measure brightness and chromaticity.. Since the focal length of CS200 was $296 \mathrm{~mm}$-limitless, I had limited distance to a Tablet PC screen at up to $60 \mathrm{~cm}$ when measuring the chromaticity of screen. This experiment was conducted to identify whether the features of chromaticity were identical when a film overlay was applied to papers like books and IT devices like smart phones. Measured color overlay coordination is presented in Figure 1(c) as CIE Lu'v'. Coordination marked on the boundaries in Figures 1 is the measurement result with overlays overlapping. Coordination marked on the inside of Figure 1 is presented as a single overlay.

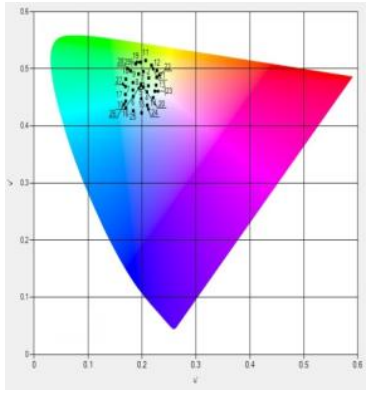

(a)
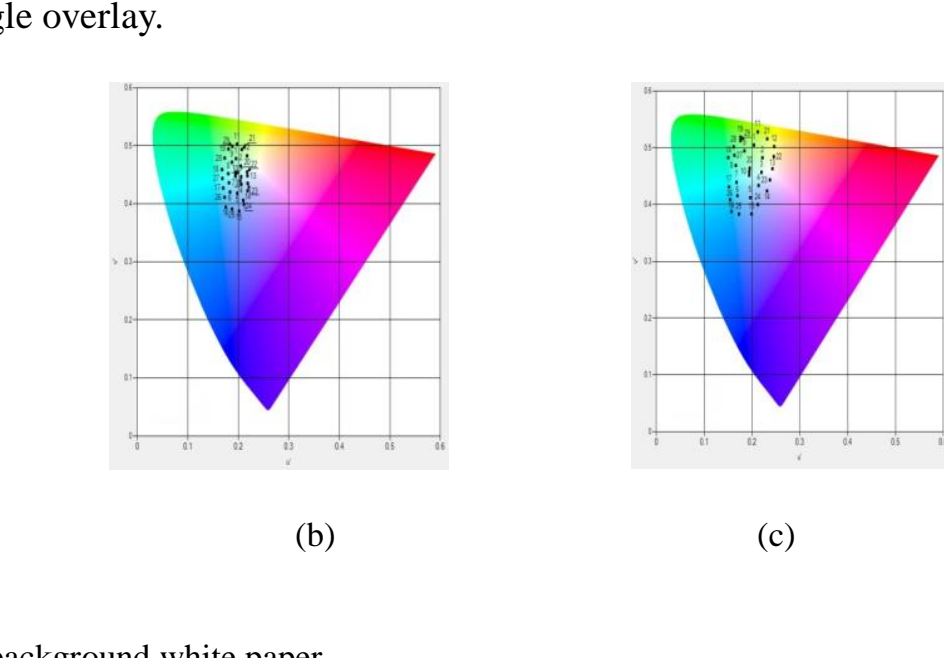

(c)

(a) Intuitive Overlays

(b) Intuitive Overlays with background white paper

(c) Intuitive Overlays with uhite backgroand of Galaxy Tab 10.1

Figure 1. Measurement Results of u'v' Chromaticity

\section{Design and Implementation}

The first operational requirement of mobile overlay in the Android operating system is the color of overlay selected in the early stage shall operate commonly in any screen. The second requirement is the mobile overlay shall not affect any screen working in a smart phone or tablet PC and applications that users interface with the exception of visual effect. Third, the mobile overlay shall be able to change selected colors in different situations.

Unlike the Windows OS, the Android OS has only one foreground application, and the screen of a corresponding application takes full screen with the exception of a status line. Whem user run an application, it will run at foreground, and users can call for other applications or other screens within the same application [12]. These programs and screens are stored in an application stack by a system activity manager. Since virtual colored overlay must be displayed continuously even when other applications or screens are running, it cannot be implemented as an activity which must use foreground activity. Services are the activity that runs without direct interactions with users. Therefore, if mobile overlay is implemented as services, it can be activated without giving effect to screens or application that users interface with. Services can be implemented in both background and foreground. In the case where background service is used, when the memory capacity of an applicable smart phone or tablet PC is not sufficient for the requirements of an operating application, an operating system forces the services to shut down. To prevent this, foreground service is required.

In foreground service, only a notification of "currently activating" is displayed (status bar at the top of the Android screen). Users can check if an applicable service is currently 
operating and they can shut down an operating system when necessary. The way to start a foreground service is to use startForeground, stopForeground, and internal method of service class. This research used services in order to operate an application all the time, implementing mobile colored overlay at the top window that is used in a screen, and not to effect interaction among currently operating applications. The function of executing or stopping virtual colored overlay service is implemented as an activity. When this application is first used, select the color suitable for users before activating overlay services with the corresponding color. Android allows multiple windows. How the window is displayed on the screen in Android is presented in Figure 2. As illustrated in the figure, when an overlay runs at the top window via background service, overlay is always on the screen regardless of which screen or app is used. In order to insert generated overlay at the top window, transfer users' touch to a displayed screen via overlay, and to keep overlay transparent, you need to set a layout parapheter of the window manager as seen in List 1 and register an overlay view in window via addview method. Addview method shall obtain permission from manifest and serfice ata later stage.

\section{Figure 2. Example of Android Screen Composed by Multiple Windows}

\section{Test and Result}

In order to verify the chromaticity of implemented mobile colored overlay, I compared the measurement value of chromaticity by applying mobile colored overlay to the white background screen of a display unit with the color value obtained by penetrating film color overlay with spectrophotometer. For measuring chromaticity, I used CS-200 portable chroma meter by Konica Minolta and for measuring transmitted light, I used CM-3600d by Konica Minolta. For the tablet PC, I used Samsung Galaxy Tab 10.1. CS-200 could measure brightness and chromaticity. During actual measurement, surrounding lightings were all shut down and all light sources were blocked. Measured color overlays coordination on Galaxy tablet 10.1 with chroma meter was presented in Figure 3 as CIE Lu'v'. Each number in the figure was the number of each overlay sample with 1-10 being single color, 11-20 being single overlapped color, and 21-29 being surrounding overlapped color numbers. Galaxy Tab 10.1 showed light purple with weak background, while the screen brightness against the white background was $394 \mathrm{~cd} / \mathrm{m}^{2}$. The differences of chromaticity were the differences of CIE Lu'v' coordination except the value of brightness and are presented as $\Delta \mathrm{u}^{\prime}$ and $\Delta \mathrm{v}^{\prime}$. Average and standard deviation of color differences $\left(\Delta E^{\prime}{ }^{\prime} v^{\prime}\right)$ between actual film overlay and mobile colored overlay were 0.022575 and 0.006654 , respectively, which was not a big deviation to be distinguished visually. Reproducibility of chromaticity value of actual CM3600d was 0.02 on the basis of standard deviation $\Delta \mathrm{E}^{*} \mathrm{ab}$, and the precision of chromaticity of chroma meter 
CS-200 was \pm 0.002 on $\mathrm{L}_{\mathrm{v}} \mathrm{xy}$ coordination. Therefore, the measured differences were at a negligible level. Examples of application of implemented mobile colored overlay app to mobile phones are presented in Figure 5. Overlay operates as a service and works in a proper way without interfering with a currently operating app.

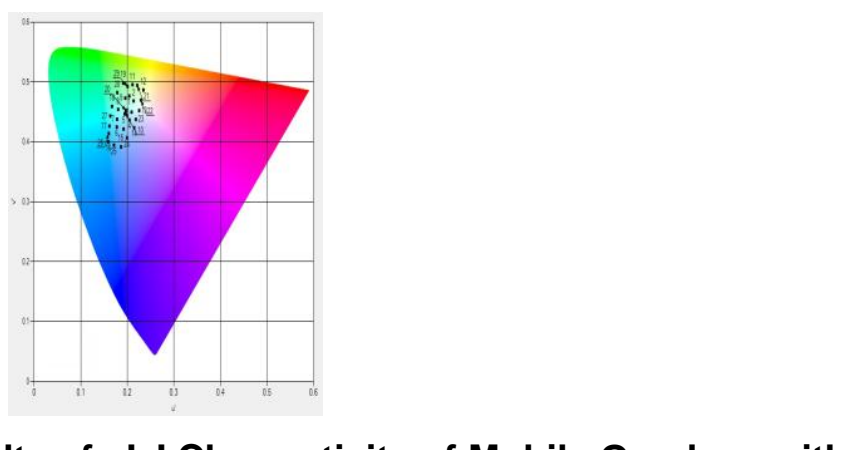

Figure 3. Measurement Results of u'v' Chromaticity of Mobile Overlays with White Background in Calaxy Tab 10.1

Figure 4. Difference of chromaticity between Mobile Overlays and Intuitive Overlays in Galaxy Tab 10.1

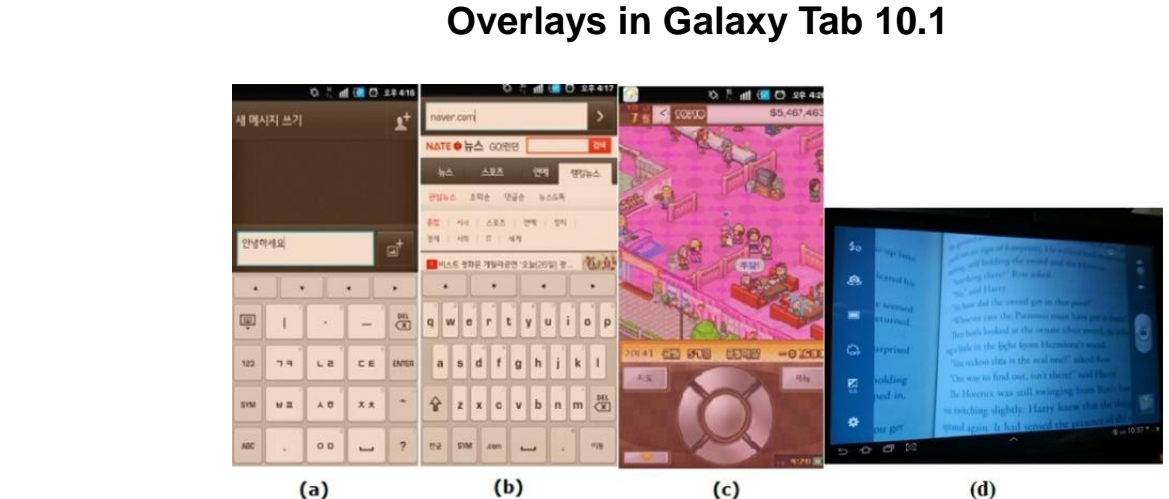

(a) Web browser sample (b) text message sample

(c) Graphic game sample (d) external text view in paper book

\section{Figure 5. Application Samples of Mobile Overlays}

\section{Conclusion}

This study implemented a mobile colored overlay app that can be used in a tablet PC operating on Android OS. This app had the effect of mitigating or removing visual stress like 
scotopic seizure that was regulated in the Korean Web Accessibility Guidelines 2.0 and Mobile Application Accessibility Guidelines. For the detailed color presentation, I implemented a single color, overlapped overlay of a single color and overlapped overlay of a neighbor color that were used in an existing film's overlay. To determine the correct original chromaticity and alpha value of a mobile overlay, color information, transmissivity of Intuitive Overlays, and the existing film overlay were measured. The alpha value was estimated to apply to mobile colored overlay through a relationship setting of alpha values. I found that if an optimized color selection test resulting in an existing film overlay was to be used in mobile overlay and existing colors whose effects were verified with those with visual stress empirically were to be implemented in mobile colored overlay, chromaticity/measured in reflect mode with white paper background instead of transmit mode should be used. I measured the chromaticity of mobile overlay on the display of a tablet PC with chroma meter and found that it was almost identical with the case that film overlay was applied to printed books.

Implemented overlay can be used in an application program such as a computer game, whose graphic object is operated by a mouse. The overlay is easy to use in the web browser application environment with a conple combination of list box selections and button controls, which can directly use colors selected from the WRRT result.

\title{
References
}

[1] Web Contents Accessibility Guidelines (WCAG) 2.0, hitp://www.w3.org/TR/WCAG20.

[2] Korean Web Content Accessibility Guidelines 2.0, Korean Telecommunication Technology Association, (2009).

[3] Mobile Application Accessibility Guidelines, MOPÂS proclamation No. 2011-38, Korean Ministry of Public Administration and Security, (2011).

[4] E. E. Schmidt, Chairman of Google "Speed, study Korea", Korea Joonang Daily, (2012) February 21.

[5] O. Mears, "Figure/ground, brightness, contrast and reading disabilities", Visible Language, vol. 14, (1980), pp. 13-29.

[6] H. Irlen, "Successful treatment of Learning Disabilities", 1st Annual Convention of the American Psychologica Association, (1983) Anaheim California.

[7] Virtual Reading Ruler, http://『yw.crossboweducation.com/.

[8] S. H. Park, H. I. Choi and Y. G. Jang, "An Implementation of Virtual Colored Overlay to Reduce Visual Information Processing Deficit", Journal of Rehabilitation Research, vol. 12, no. 1, (2008), pp. 138-159.

[9] aaLuminate, http://www aardwearing-software.com/aaLuminate/Welcome.html.

[10] Y. G. Jang, "Virtual Colored Overlays in Mobile Devices for People with Visual Stress to Improve Readability", Advanced Science and Technology Letters, vol. 43, (2013), pp. 6-9.

[11] Intuitive overlays dispensing packs, http://www.ioosales.co.uk/html/practice/eye05E.html.

[12] Ed Burnette Hello, Android: Introducing Google's Mobile Development Platform", $1^{\text {st }}$ Edition, Pragmatic Bookshelf. (2009).

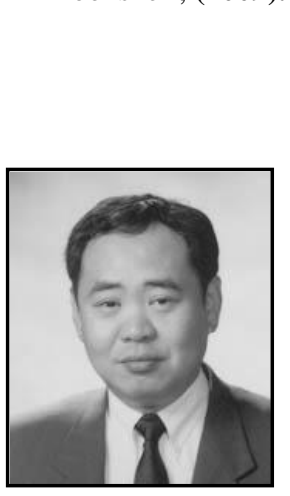

\begin{abstract}
Author
Young Gun Jang, He received the B.E., M.S., and Ph.D. degrees from Inha Univ. in 1980, 1991 and 1995, respectively. He worked as a research engineer at the Agency of Defense Development (from 1979), a senior research engineer at the Daewoo Heavy Industry Inc.(from 1983), a senior engineer at the Institute of Advanced Engineering(1995-1996), visiting researcher at the University of California, Davis (2003-2004). He has been a professor at the Dept. of Computer \& Information Engr. in Chongju Univ. from 1996. His research interest includes HCI, Assistive Technology, Security, Intelligent Robot, and Intelligent Web Information Processing.
\end{abstract}

